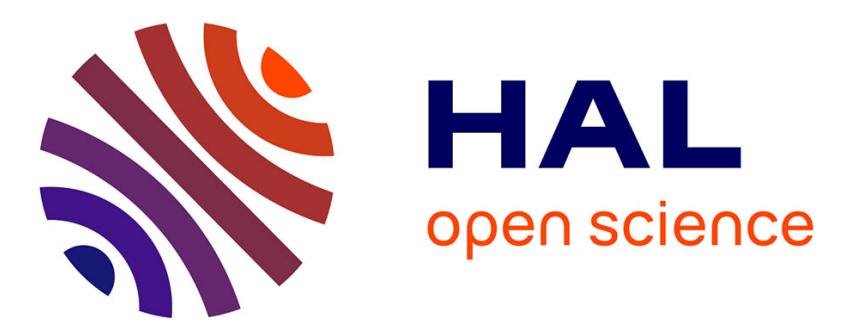

\title{
Influence of Source Propagation Direction and Shear Flow Profile in Impedance Eduction of Acoustic Liners
}

Remi Roncen, Estelle Piot, Fabien Mery, Frank Simon, Michael G. Jones, Douglas M. Nark

\section{- To cite this version:}

Remi Roncen, Estelle Piot, Fabien Mery, Frank Simon, Michael G. Jones, et al.. Influence of Source Propagation Direction and Shear Flow Profile in Impedance Eduction of Acoustic Liners. 25th AIAA/CEAS Aeroacoustics Conference, May 2019, DELFT, Netherlands. hal-02194989

\section{HAL Id: hal-02194989 \\ https://hal.science/hal-02194989}

Submitted on 26 Jul 2019

HAL is a multi-disciplinary open access archive for the deposit and dissemination of scientific research documents, whether they are published or not. The documents may come from teaching and research institutions in France or abroad, or from public or private research centers.
L'archive ouverte pluridisciplinaire HAL, est destinée au dépôt et à la diffusion de documents scientifiques de niveau recherche, publiés ou non, émanant des établissements d'enseignement et de recherche français ou étrangers, des laboratoires publics ou privés. 


\title{
Influence of Source Propagation Direction and Shear Flow Profile in Impedance Eduction of Acoustic Liners
}

\author{
R. Roncen* , E. Piot, F. Mery and F. Simon, \\ ONERA/DMPE, Université de Toulouse, F-31055, Toulouse, France \\ M. G. Jones and D. M. Nark \\ NASA Langley Research Center, Hampton, VA 23681-2199, U.S.A
}

\begin{abstract}
The acoustic impedance of liners is a key parameter for their design, and depends on the flow conditions, i.e., the sound pressure level and the presence of a grazing flow. The surface impedance of a locally reacting liner is defined as a local intrinsic property relating the acoustic pressure to the normal acoustic particle velocity at the liner surface. Impedance eduction techniques are now widely used to retrieve the impedance of liners in aeroacoustic facilities in the presence of a shear grazing flow. While surface impedance is intrinsic by definition, the educed impedance has recently been shown to depend on the direction of the incident waves relative to the mean flow. Different studies have investigated this issue by considering different acoustic propagation models used in the eduction process in the hope of matching the educed values. The purpose of the present work is to continue the previous investigations by evaluating the influence of the shear flow profile on the educed impedance, while considering a Bayesian inference process in order to evaluate the uncertainty on the educed values. The identified uncertainties were not able to totally account for the observed discrepancies between educed impedances.
\end{abstract}

\section{Nomenclature}

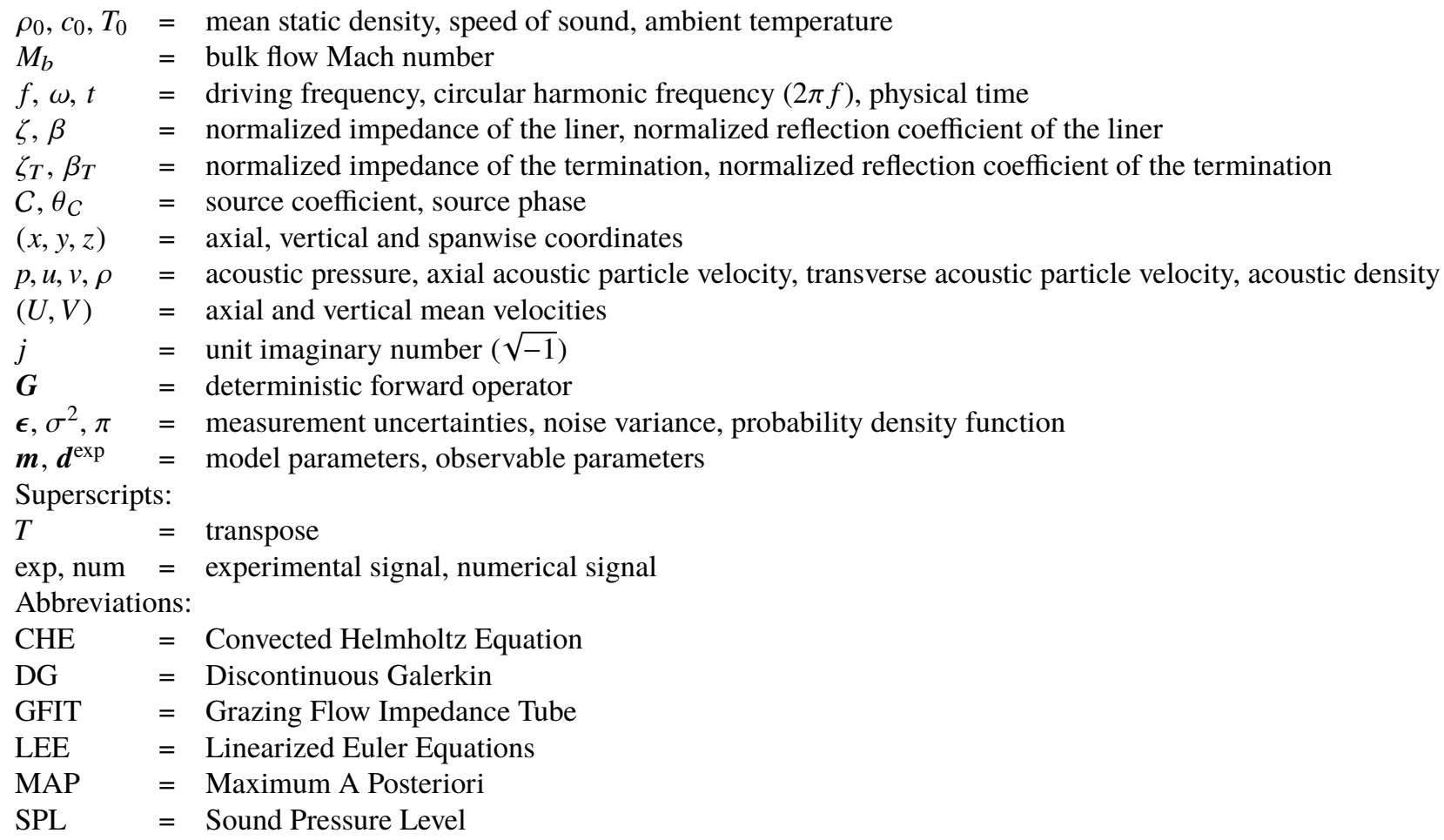




\section{Introduction}

Aircraft engines are one of the dominant noise sources for subsonic aircraft, resulting in a lot of research toward the noise reduction inside the engine nacelle. In current turbofan engines, the inlet, bypass and exhaust parts of the nacelle are lined with acoustic liners. Liners consist essentially of a perforated plate backed by an air cavity, and have a quarter-wavelength behavior.

The property employed to represent the locally reacting liner's acoustic behavior is the impedance. It relates the acoustic pressure to the normal acoustic particle velocity. The impedance is defined as an intrinsic parameter associated with a given liner configuration. However, estimates of the impedance from experimental measurements depend on the sound pressure level and the shear grazing flow velocity and profile. The accurate knowledge of the influence of a complex flow on the impedance is of prime importance when it comes to liner design and optimization [13, 16]. It is equally important to be able to educe the impedance value of a liner subject to a shear grazing flow, which is known to complicate the measurement.

A wide number of different methods have been developed to educe the impedance, broadly separated into two different classes: straightforward and inverse methods. Straightforward methods use a measurement of an axial propagation constant in conjunction with the impedance boundary condition to educe the impedance directly, without iterations. Recent examples of developments in this field are found in Refs. [2, 4, 17]. Indirect eduction methods [3, 11] are now widely used to estimate the acoustic impedance of liner materials in the presence of a grazing flow. These methods usually consist of measuring the acoustic pressure on the wall opposite the liner and using a minimization method, where the acoustic pressure fields obtained by solving partial differential equations coupled with an impedance boundary condition are compared with experimental measurements.

While intrinsic by definition, the educed impedance has recently been shown to depend on the direction of the incident waves relative to the mean flow [12], i.e., upstream or downstream sources. This observed behavior has led to questions about the assumptions underlying the current eduction methods [7, 14, 15].

The purpose of the present study is to investigate the influence of the shear grazing flow profile on the impedance eduction process for upstream and downstream sources. In addition, an uncertainty quantification based on a Bayesian inference process is performed to evaluate the uncertainties on the educed impedance for both source locations. The general inference strategy and the experimental set up are recalled in Sec. III. The eduction results are presented in Sec. III] Concluding remarks are laid out in Sec. IV.

\section{Statistical Inference Strategy}

This section presents the necessary elements to the impedance eduction strategy. First, the general geometry of a typical aeroacoustic bench used for impedance eduction is recalled. The direct problem, consisting of solving the Linearized Euler Equations (LEE), is then introduced. Finally, the Bayesian inference method used in this work is detailed.

\section{A. Experimental set up}

The raw acoustic data used in this work were obtained by NASA and shared through a NASA-ONERA cooperation on liner eduction techniques. The Grazing Flow Impedance Tube (GFIT) was selected to evaluate the acoustic liners in the presence of a grazing flow. Details about the GFIT dimensions can be found for instance in Ref. [7, II.A]. Different flow ducts are used around the world for liner eduction [1, 2, 11], but the concepts remain quite similar. Acoustic plane waves are generated in the duct using drivers, over a frequency range of 400 to $3000 \mathrm{~Hz}$ in $200 \mathrm{~Hz}$ increments. In the present study, the acoustic source can either be located upstream or downstream of the liner sample. Different nonintrusive measurement devices can then be added to access information on the acoustic fields inside the duct, such as microphones on the wall opposite the liner. The measured acoustic fields can then be compared to the numerical fields obtained by solving the set of equations controlling the acoustic wave propagation in the duct, as detailed in Sec. II.B. A schematic of an aeroacoustic bench is shown in Fig. 1 in the case of an upstream source. The unknown parameters are the source coefficient $C$, the liner normalized impedance $\zeta$ and the termination impedance $\zeta_{T}$. In the case of a downstream located source, the positions of $C$ and $\zeta_{T}$ are reversed in the figure.

\section{B. Direct problem}

To perform an inverse identification of the liner impedance $\zeta$, it is necessary to link the parameters to be identified ( $C$, $\zeta$ and $\zeta_{T}$ ) to the observed measurement (the pressure field on the wall opposite the liner). To do so, the two-dimensional 


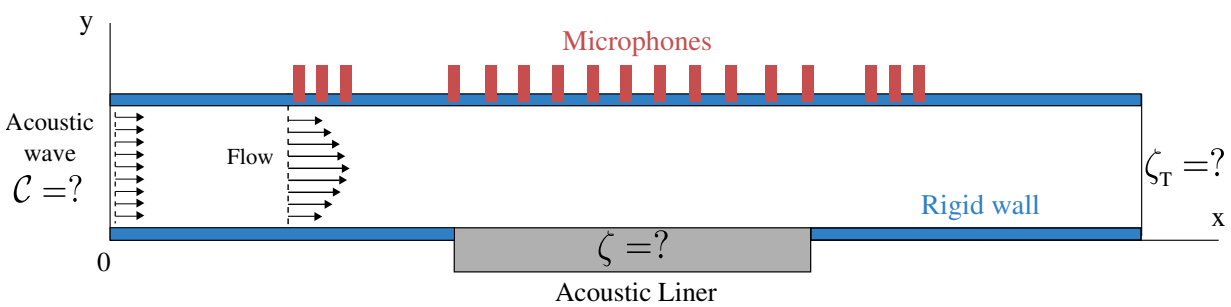

Figure 1 Schematics of an aero-acoustic bench for liner impedance eduction.

time-harmonic LEE are solved using a Discontinuous Galerkin (DG) scheme, where the parameters of interest are integrated into the different boundary conditions. The LEE, written in nonconservative form with an $e^{j \omega t}$ time dependence and assuming homentropic flow, are given by

$$
\mathbf{L} \varphi=0
$$

where

$$
\mathbf{L} \boldsymbol{\varphi}=\mathrm{j} \omega \varphi+\boldsymbol{A}_{x} \frac{\partial \varphi}{\partial x}+\boldsymbol{A}_{y} \frac{\partial \varphi}{\partial y}+\boldsymbol{B} \boldsymbol{\varphi}
$$

and

$$
\boldsymbol{A}_{x}=\left(\begin{array}{ccc}
U & 0 & c_{0} \\
0 & U & 0 \\
c_{0} & 0 & U
\end{array}\right), \boldsymbol{A}_{y}=\left(\begin{array}{ccc}
V & 0 & 0 \\
0 & V & c_{0} \\
0 & c_{0} & V
\end{array}\right), \boldsymbol{B}=\left(\begin{array}{ccc}
\partial_{x} U & \partial_{y} U & 0 \\
\partial_{x} V & -\partial_{x} U & 0 \\
0 & 0 & 0
\end{array}\right) .
$$

In the above equations, $\varphi=\left(u, v, \frac{p}{\rho_{0} c_{0}}\right)$. $p$ is the acoustic pressure, and $u, v$ represent the acoustic perturbations around the shear mean flow of axial and transverse velocities $U$ and $V$, respectively. Due to the homentropy condition, the energy equation is replaced by the state equation $p=c_{0}^{2} \rho$, where $\rho$ is the acoustic density.

Since we consider a shear mean flow profile, the velocity at the walls is 0 , and there is no need to implement an Ingard-Myers boundary condition. The classical impedance boundary condition is used to relate the pressure to the normal component of the velocity at the liner surface as

$$
p= \pm \rho_{0} c_{0} \zeta v
$$

where the sign is chosen depending on whether the upper or lower wall is considered. To avoid any singularities in the rigid wall case, the boundary condition (4) is expressed as a function of the normal incidence reflection coefficient $\beta=(\zeta-1) /(\zeta+1)$. This leads to the following boundary condition matrix:

$$
\boldsymbol{M}_{\beta}=\frac{1}{2}\left(\begin{array}{cc}
(\beta+1) \boldsymbol{n} \otimes \boldsymbol{n} & (\beta-1) \boldsymbol{n} \\
-(\beta+1) \boldsymbol{n}^{t} & (1-\beta)
\end{array}\right),
$$

where $\boldsymbol{n}$ is the local normal vector and $\otimes$ the tensor product. On the rigid walls, the reflection coefficient $\beta$ is then taken as 1 .

At the source plane, the state vector $\varphi$ is taken to be equal to $C \varphi_{0}$, where $\varphi_{0}$ is the plane-wave solution in a rigid duct with uniform flow Mach number $M_{b}$. It corresponds to the bulk Mach number of the actual shear flow $U(y)$, calculated by averaging the measurements performed at the center span and at 3 different $x$ locations. As this plane-wave solution is chosen with an arbitrary amplitude of 1 , the complex coefficient $C$ determines the source amplitude and phase. At the inlet boundary conditions, the two enforced boundary conditions required by the 2D LEE are a specified pressure $p$, and $v=0$.

A Discontinuous Galerkin (DG) scheme is chosen to solve Eq. (1) and the associated boundary conditions. In this method, discontinuities are allowed at the interface between two elements. An example of how such a method can handle discontinuities at hard-soft wall interfaces is found elsewhere [8, 9]. To ensure the connection between elements and to apply the boundary conditions, a numerical flux is defined. An upwind numerical flux is used to ensure connection between interior cells. A centered flux is used at the boundaries (see previous papers [10, 11] for more details). 
Table 1 Prior bounds for a uniform probability

\begin{tabular}{ccccccc} 
Parameter & $\mathfrak{R}(\beta)$ & $\mathfrak{J}(\beta)$ & $|\mathcal{C}|$ & $\theta_{C}$ & $\mathfrak{R}\left(\beta_{T}\right)$ & $\mathfrak{J}\left(\beta_{T}\right)$ \\
\hline$m_{i, \min }$ & -1 & -1 & 0.1 & $-\pi$ & -1 & -1 \\
$m_{i, \max }$ & 1 & 1 & 2.0 & $+\pi$ & 1 & 1
\end{tabular}

\section{Bayesian Inference for Liner Impedance Eduction}

The eduction method in this work relies on a statistical approach to inverse problems. Bayes' theorem is used to relate an observation with uncertainties (the pressure measurement) to the variables of interest. The parameters of interest $\boldsymbol{m}=\left(\mathfrak{R}(\zeta), \mathfrak{J}(\zeta),|C|, \theta_{C}, \mathfrak{R}\left(\zeta_{T}\right), \mathfrak{J}\left(\zeta_{T}\right)\right)$ are the realizations of random variables, and their posterior probability density is the quantity to be found. It encompasses all the knowledge that has been extracted from the observation $\boldsymbol{d}^{\exp }$. The posterior probability density $\pi\left(\boldsymbol{m} \mid \boldsymbol{d}^{\exp }\right)$ is written as

$$
\pi\left(\boldsymbol{m} \mid \boldsymbol{d}^{\exp }\right) \propto \overbrace{\pi\left(\boldsymbol{d}^{\mathrm{exp}} \mid \boldsymbol{m}\right)}^{\text {Likelihood }} \overbrace{\pi(\boldsymbol{m})}^{\text {Prior }} .
$$

The likelihood function $\pi\left(\boldsymbol{d}^{\exp } \mid \boldsymbol{m}\right)$ represents the evaluation of the direct problem described in Sec. II.B.

The observed data $\boldsymbol{d}^{\exp }$ is related to the model parameters through

$$
\boldsymbol{d}^{\exp }=\boldsymbol{G}\left(\boldsymbol{m}^{*}\right)+\boldsymbol{\epsilon}
$$

with $\boldsymbol{m}^{*}$ unknown, where $\boldsymbol{G}$ is the deterministic forward observation operator. It encompasses the full resolution of the LEE by a DG scheme, and the extraction of the pressure field on the wall opposite the liner at the measurement locations. $\boldsymbol{\epsilon}$ accounts for measurement uncertainties, with components of $\boldsymbol{\epsilon}$ being independent and identically distributed random variables of density $\pi_{\epsilon}$. For brevity of notation, we shall write the array's $i^{\text {th }}$ components $d_{i}^{\text {exp }}$ and $G_{i}(\boldsymbol{m})$. The likelihood can be written, assuming $\boldsymbol{\epsilon}$ follows a normal distribution, as

$$
L(\boldsymbol{m})=\prod_{i=1}^{n_{d}} \frac{1}{\sqrt{2 \pi \sigma_{i}^{2}}} \exp \left(-\frac{\left|d_{i}^{\exp }-G_{i}(\boldsymbol{m})\right|^{2}}{2 \sigma_{i}^{2}}\right),
$$

where $\sigma_{i}^{2}$ denotes the measurement variance.

In the present article, $\sigma_{i}^{2}$ has been estimated for the pressure measurements by assuming a $\pm 0.5 \mathrm{~dB}$ uncertainty in measured amplitude and $\mathrm{a} \pm 1^{\circ}$ uncertainty in measured phase. The error was assumed constant over the different microphone locations. It can be argued that at locations where the acoustic signal is weak relative to the flow noise, the error would be increased.

The prior probability $\pi(\boldsymbol{m})$ contains statistical information obtained on the model parameters independently of the experimental data used during the inference. It reflects the user's insight on the problem and known physical constraints. When no a priori information is available, a homogeneous probability density of large range is appropriate. In the present work, we first assume prior independence between the unknown parameters, setting

$$
\pi(\boldsymbol{m})=\prod_{i=1}^{n_{m}} \pi_{i}\left(m_{i}\right)
$$

where $\pi_{i}$ follows a uniform distribution with bounds given in Table 1 Due to the possible problem ill-posedness, a Gaussian prior distribution should be used for the termination impedance value (which has been measured directly).

The posterior density provides the full distribution of the parameters of interest, from which point estimates such as the mean or median can be extracted. The parameter value that maximizes $\pi\left(\boldsymbol{m} \mid \boldsymbol{d}^{\mathrm{exp}}\right)$, coined Maximum A Posteriori (MAP) estimate, or mode, has been selected as the reference estimate. Its value is given by

$$
\boldsymbol{m}_{\mathrm{MAP}}=\underset{\boldsymbol{m}}{\arg \max } \pi\left(\boldsymbol{m} \mid \boldsymbol{d}^{\exp }\right),
$$

or, equivalently

$$
\boldsymbol{m}_{\mathrm{MAP}}=\underset{\boldsymbol{m}}{\arg \max } \pi\left(\boldsymbol{d}^{\mathrm{exp}} \mid \boldsymbol{m}\right) \pi(\boldsymbol{m}) .
$$


For a uniform prior distribution, the MAP estimate is equivalent to the classical deterministic least square minimum. For uncorrelated prior distribution, the variances and the Pearson correlation coefficients between each random variables

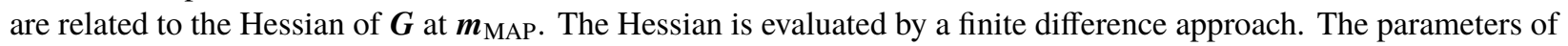
interest are assumed to have a Gaussian distribution across all the studied configurations (Ref. [6]). There is no need for an iterative MCMC procedure as was done in [6], which circumvents unnecessary calculations.

\section{Eduction Results on NASA-GFIT Test Liners}

Two different liners were tested in the GFIT, at a bulk Mach number of 0.3 . The two samples are first succinctly described, and the educed impedance is analyzed.

\section{A. Description of Test Configurations}

\section{Flow configurations}

Three different velocity profiles of decreasing shear have been considered as part of this inference study. These profiles are represented in Fig. 2, along with a fit of the experimental profile. Our profiles are not as sheared as the experimental one. Sheared flow tended to provoke the appearance of hydrodynamic modes that lead to nonphysical impedance eductions.

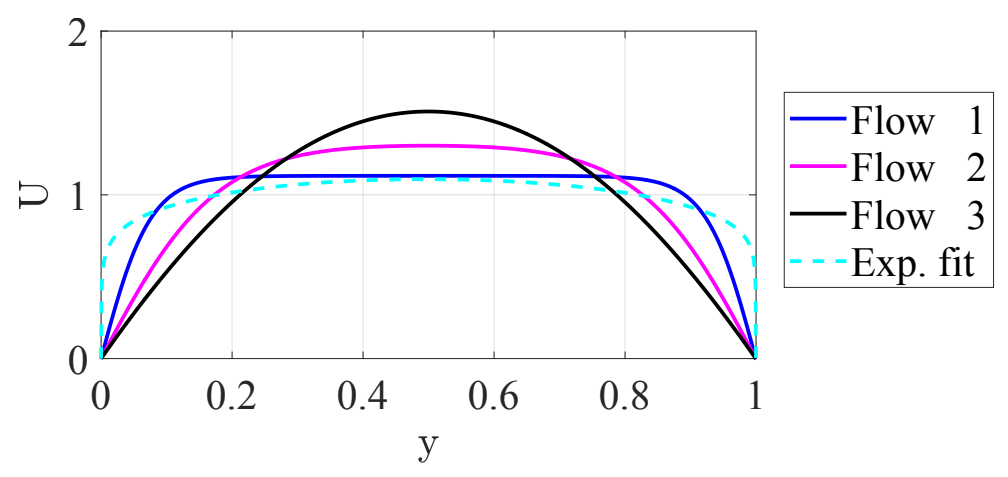

Figure 2 Flow profiles used for the impedance eduction. Velocities are normalized by the mean experimental velocity.

\section{Test liners}

The first liner is called CT57 and consists of ceramic tubes of diameter $0.635 \mathrm{~mm}$ and length $82.55 \mathrm{~mm}$. The surface porosity of the sample is 57\%. The CT57 has an impedance that is almost independent of the source SPL and of the tangential flow velocity [5]. The second liner is a standard liner made of a perforated plate of thickness $t_{\mathrm{p} . \mathrm{p}}=0.635 \mathrm{~mm}$, perforation diameter $d_{\mathrm{p} . \mathrm{p}}=0.991 \mathrm{~mm}$ and porosity $15 \%$, backed by a honeycomb cavity of depth $d_{\mathrm{c}}=38.1 \mathrm{~mm}$. The impedance of this liner is only weakly dependent on the source SPL and tangential flow velocity [5], due to its high surface porosity. A sketch of the two test liners is represented in Fig. 3

\section{B. Influence of the Flow Profile on the Educed Impedance}

\section{CT57 test liner}

Identification results for test liner CT57 are displayed in Fig. 4 To increase clarity, the error bars are omitted. The color chart corresponds to the one in Fig. 2] Eduction results of Ref.[7], obtained by solving an inverse problem relying on the Convected Helmholtz Equations ( $\mathrm{CHE}$ ), are displayed in green in Fig. 4 

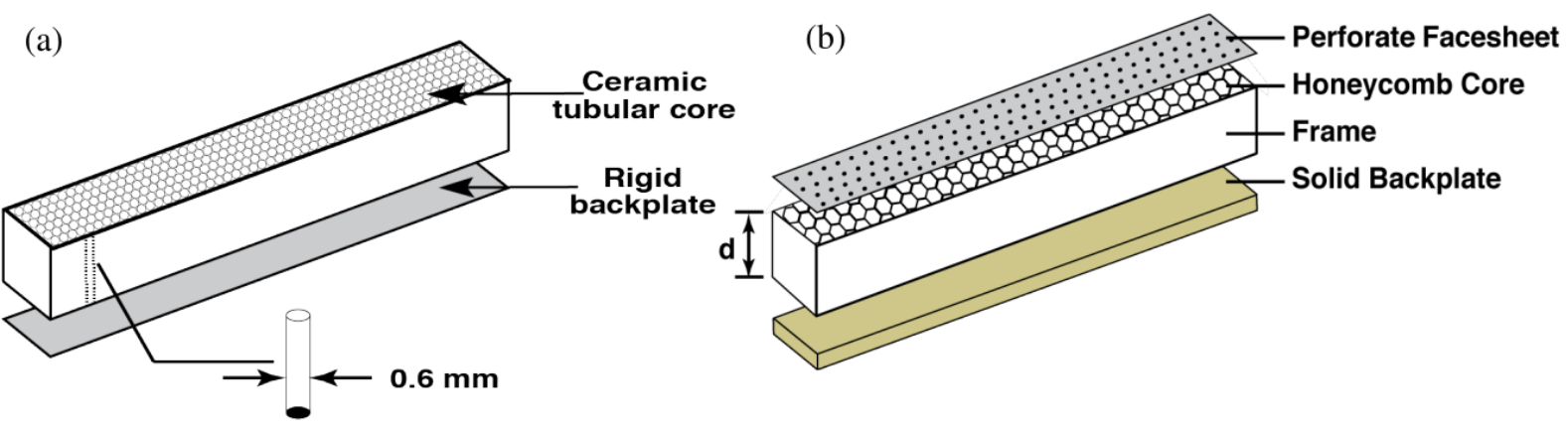

Figure 3 Sketches of CT57 and GE03 test liners (from Ref. [7]).
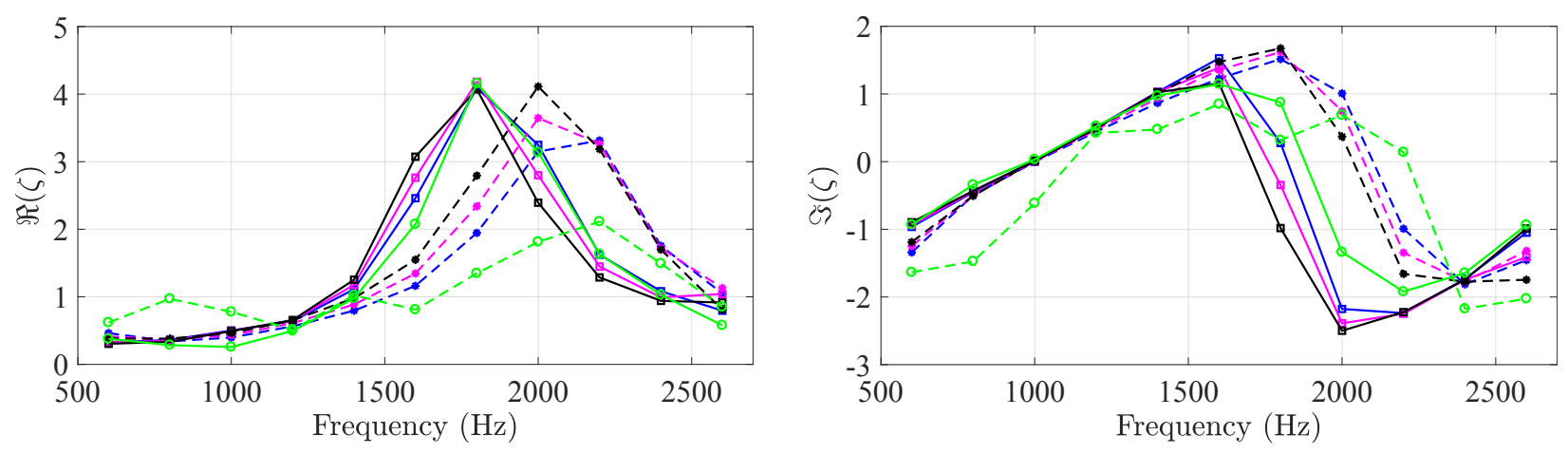

Figure 4 CT57 impedance identification at $M_{b}=0.3$. Upstream source (solid line), downstream source (dashed line). Green curves from Ref. [7] using the CHE.

The effect of changing the tangential flow profile is more marked near the antiresonance of the liner, where incidentally the spread between impedance educed from upstream and downstream configurations is the largest. We note, however, that the overall effect of changing the flow profile is similar for both source locations. An increased shear tends to reduce the resistance before the antiresonance and increases it after. As a result, the resistance curves appear shifted to lower frequencies. An increased shear tends to increase the reactance over all the antiresonance frequency range, again resulting in an apparent shift toward lower frequencies.

The CT57 is assumed to have an impedance independent from the presence of a flow [5]. The educed impedance obtained at $M_{b}=0$ is superimposed on the impedance educed with flow 1 (see Fig. 2), along with the 99\% credibility intervals, in Fig. 5. In the Bayesian sense, such an interval is interpreted as a $99 \%$ probability that the true value of a given parameter falls within the credible region. The impedance educed at $M_{b}=0$ is indeed very close to the one educed at $M_{b}=0.3$ for frequencies below $1400 \mathrm{~Hz}$. A discrepancy appears at higher frequencies, when approaching the antiresonance, but curves seem to coalesce again after $2500 \mathrm{~Hz}$. Overall, the impedance identified in the downstream configuration at $M_{b}=0.3$ is nearest to the reference impedance at $M_{b}=0$. 

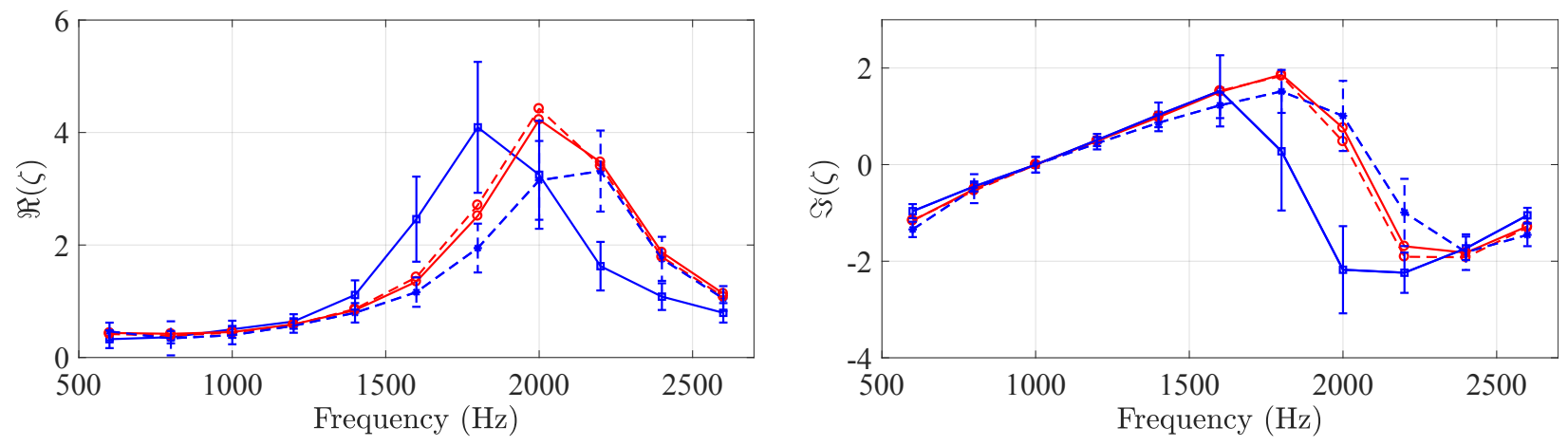

Figure 5 CT57 educed impedance at $M_{b}=0.3$. Upstream source (solid line), downstream source (dashed line). The red color is used for the values identified at $M_{b}=0$.

To understand how these differences in educed impedance reflect on the pressure at the wall opposite the liner, in comparison to the experimental data, the absolute pressure and phase are displayed in Fig. 66.7. In each figure, the 6 curves are obtained by solving the LEE using the MAP impedance educed for the 3 different flow profiles and 2 source locations. The solid and dashed lines are obtained using the impedance values educed in the upstream and downstream configurations, respectively. The color chart remains unchanged (see Fig. 2). The symbols represent the discrete observed data.
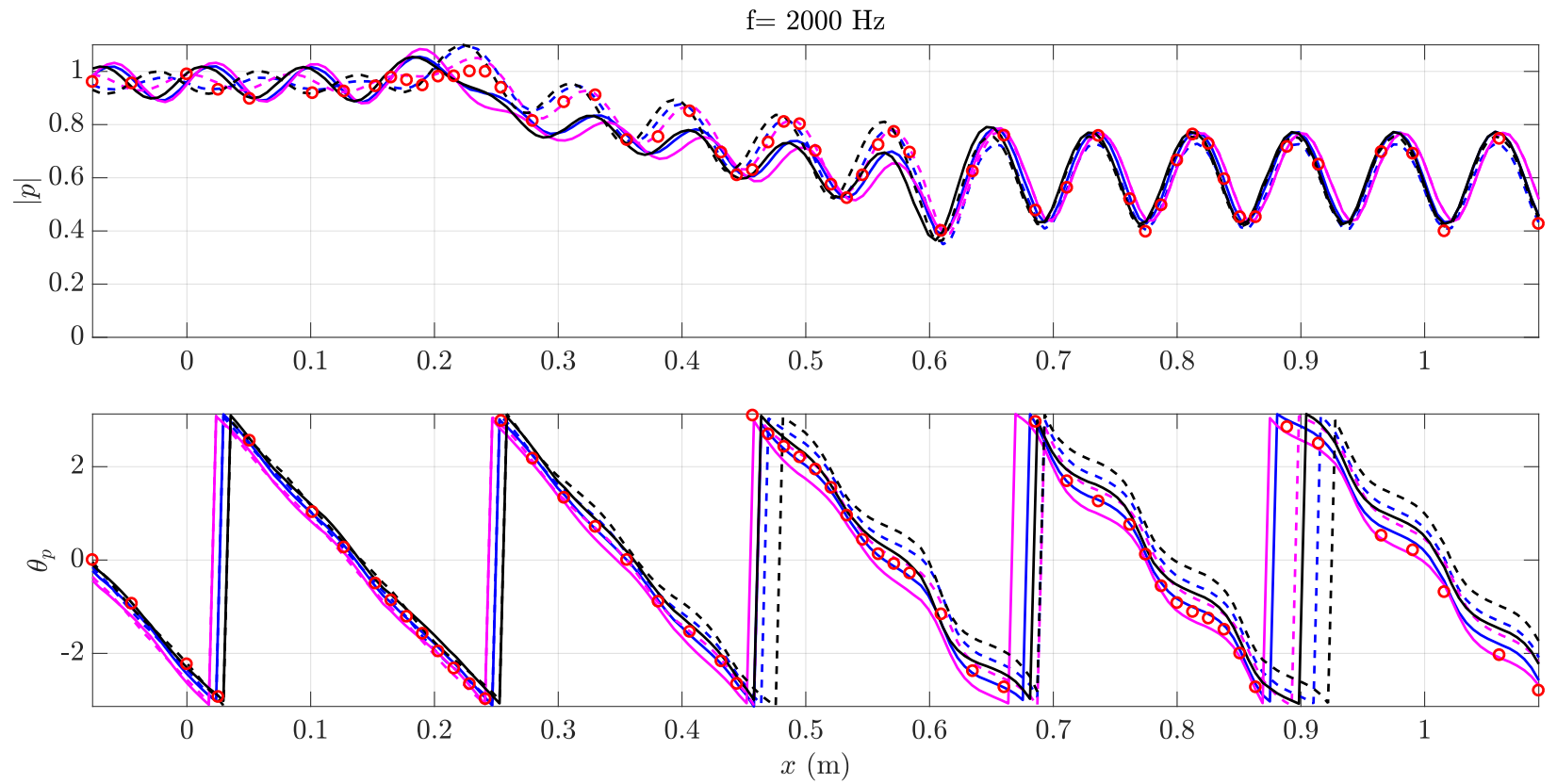

Figure 6 Wall pressure in upstream source configuration for the CT57. Upper: absolute pressure. Lower: phase. Symbols: experimental data. 

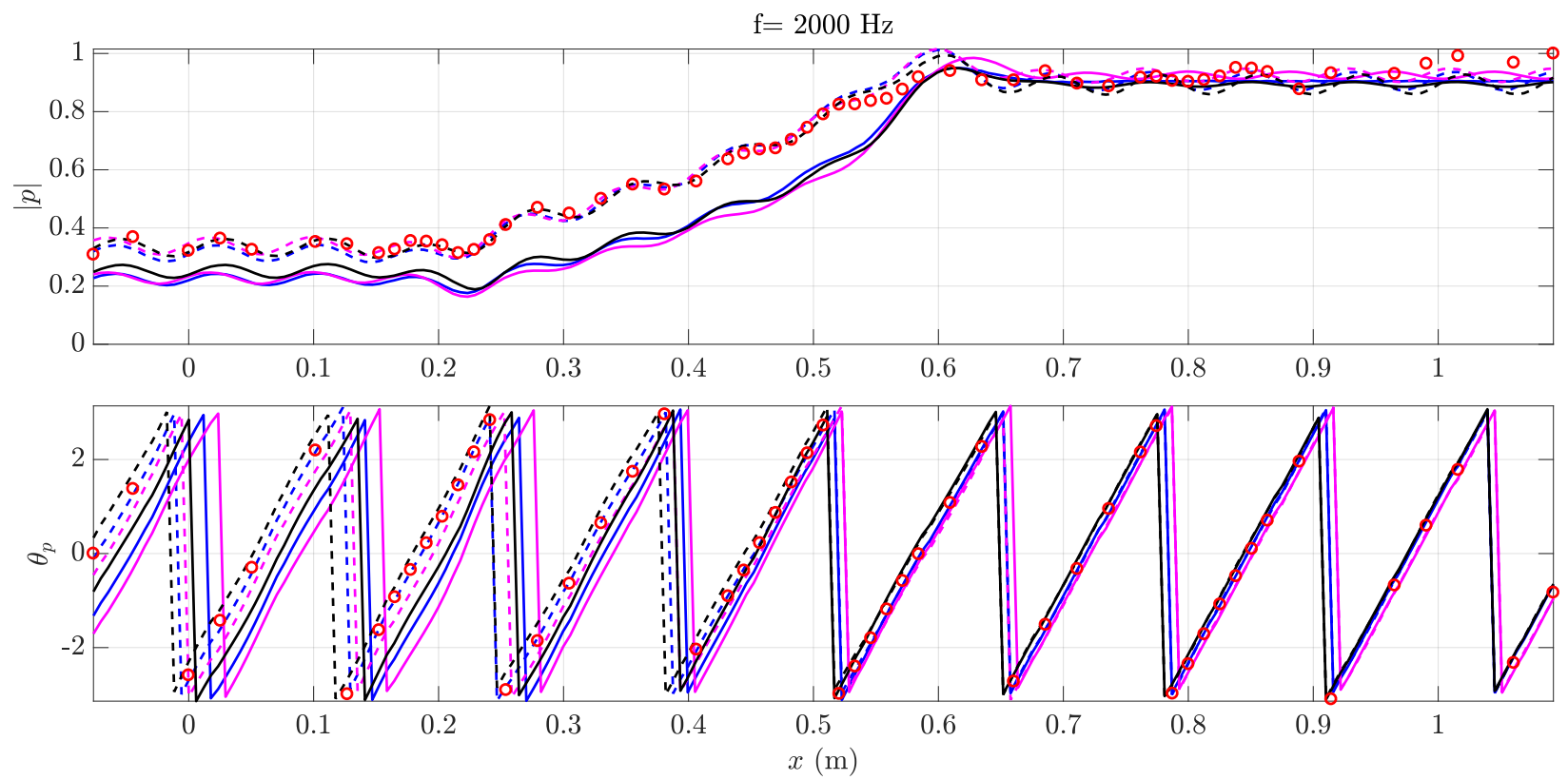

Figure 7 Wall pressure in downstream source configuration for the CT57. Upper: absolute pressure. Lower: phase. Symbols: experimental data.

For both source configurations, the influence of the flow profile is less significant than the impedance choice. Sensitivity of wall pressures to the impedance in the upstream configuration (Fig. 6) appears less marked than the one in the downstream configuration (Fig. 77). In Fig. 7, a more visible change between curves is observed when the upstream educed impedance is used instead of the downstream one. A quantitative analysis of the spread between curves is performed by assigning to each curve its cost function value (minus log of the likelihood) in Table 2, normalized by the minimum value obtained (0.13). Lower values of the cost function correspond to a better fit between experimental and numerical signals.

Table 2 Comparison of the cost function values associated with Figs. 6 7, for the CT57 at $2000 \mathrm{~Hz}$.

\begin{tabular}{ccccccc} 
& \multicolumn{3}{c}{ Upstream Identification } & \multicolumn{2}{c}{ Downstream Identification } \\
\hline & Flow 1 & Flow 2 & Flow 3 & Flow 1 & Flow 2 & Flow 3 \\
Educed impedance & $3.2-2.2 \mathrm{j}$ & $2.4-2.5 \mathrm{j}$ & $2.8-2.4 \mathrm{j}$ & $3.1+1 \mathrm{j}$ & $4.1+0.4 \mathrm{j}$ & $3.6+0.7 \mathrm{j}$ \\
Cost function in upstream conf. & 1.7 & 7.8 & 8.5 & 14.2 & 7.3 & 33.8 \\
Cost function in downstream conf. & 12.6 & 23.5 & 9.9 & 1 & 6.4 & 6.2
\end{tabular}

The fits obtained with a more sheared flow (flow 1, see Fig. 2p appear to be of superior quality than the ones with less sheared profiles, in both source configurations. This shows the necessity to consider the true shear of the flow profile in future eductions. The signals obtained in the downstream configuration show a lower cost function value than the one obtained in the upstream configuration. This gives more credit to the identified value of the impedance in the downstream configuration.

\section{GE03 test liner}

Identification results for test liner GE03 are displayed in Fig. 8. To increase clarity, the error bars are omitted. The color chart corresponds to the one on Fig. 2, and the y-axis scale is the one used for the CT57 in Fig. 4, to facilitate the comparison. 

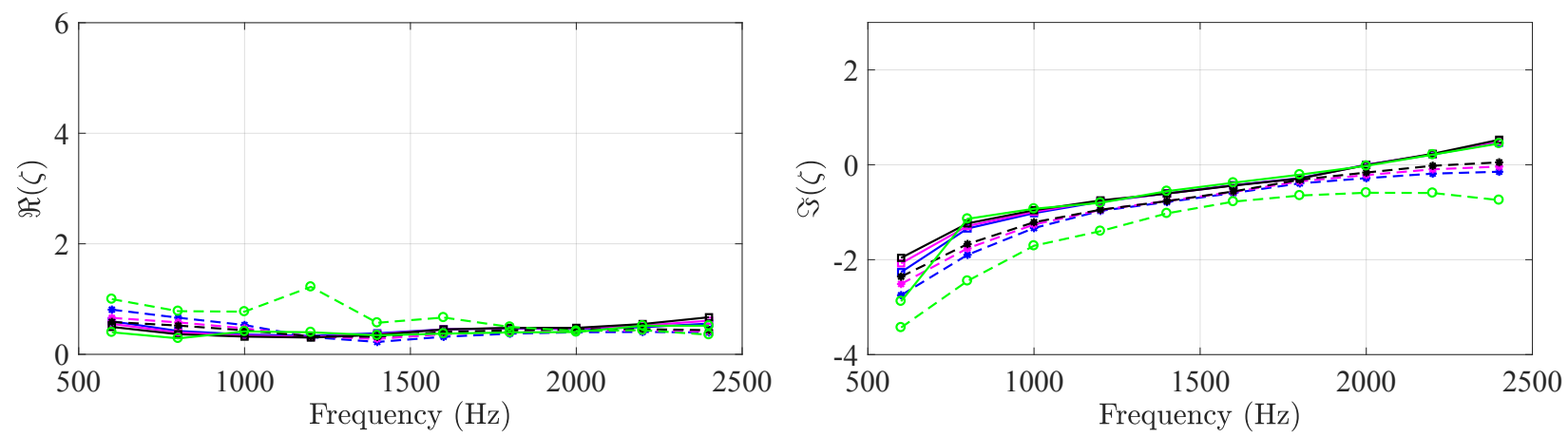

Figure 8 GE03 impedance identification at $M_{b}=0.3$. Upstream source (solid line), downstream source (dashed line). Green curves from Ref. [7] using the CHE.

We note that the educed impedance, especially the resistance, shows only a small range of values, compared to the ones identified for the CT57, and the curves are almost indiscernible. This behavior is similar to the one displayed by the CT57 at frequencies below the resonance frequency. As with the CT57 liner, the influence of the tangential flow profile on the educed liner impedance is comparable for both source locations. An increase in resistance is observed as the tangential flow velocity profile is more sheared, followed by an inverse trend after a certain frequency $(\approx 1200 \mathrm{~Hz})$. This switch in behavior does not occur anymore at the antiresonance frequency (which is not observed for this material). In addition, the reactance seems to always decrease as the shear is increased, a tendency opposed to the one noted for the CT57 at frequencies below the antiresonance.

The credibility intervals associated to the impedance educed with the flow 1 (see Fig. 2) are represented on Fig. 9
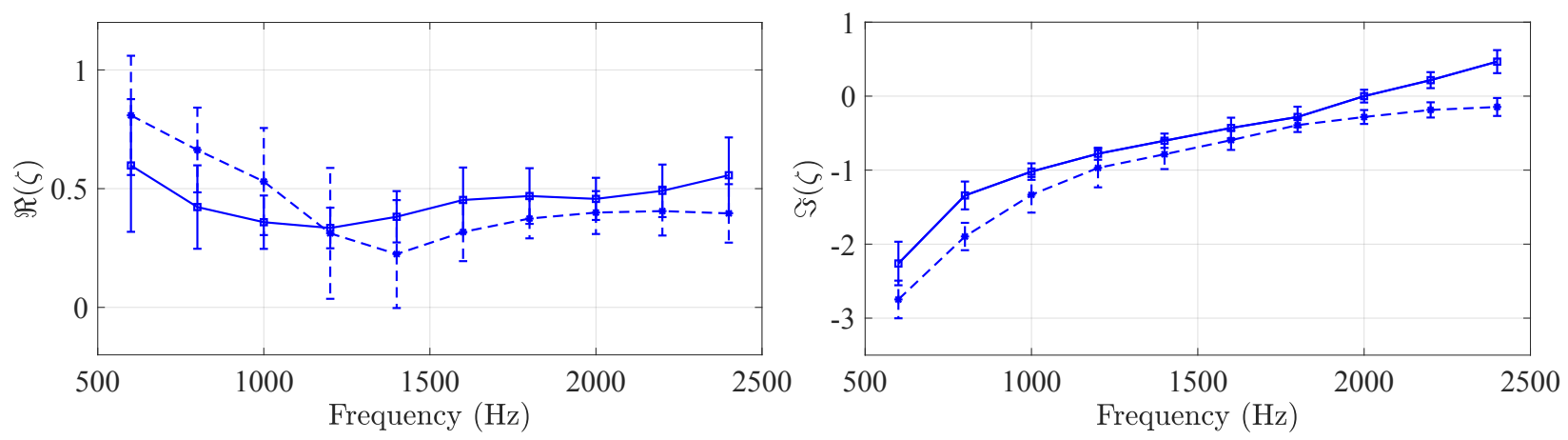

Figure 9 GE03 impedance identification at $M_{b}=0.3$. Upstream source (solid line), downstream source (dashed line).

Overall, the credibility intervals are similar for both configurations. While an overlap of the resistance is observed, it is not the case for the reactance. The uncertainties in impedance eduction associated with a $\pm 0.5 \mathrm{~dB}$ uncertainty in measured amplitude and $\mathrm{a} \pm 1^{\circ}$ uncertainty in measured phase are not able to account for the observed difference.

The absolute pressure and phase are displayed in Fig. 10 11, at $2000 \mathrm{~Hz}$, close to the liner resonance. 

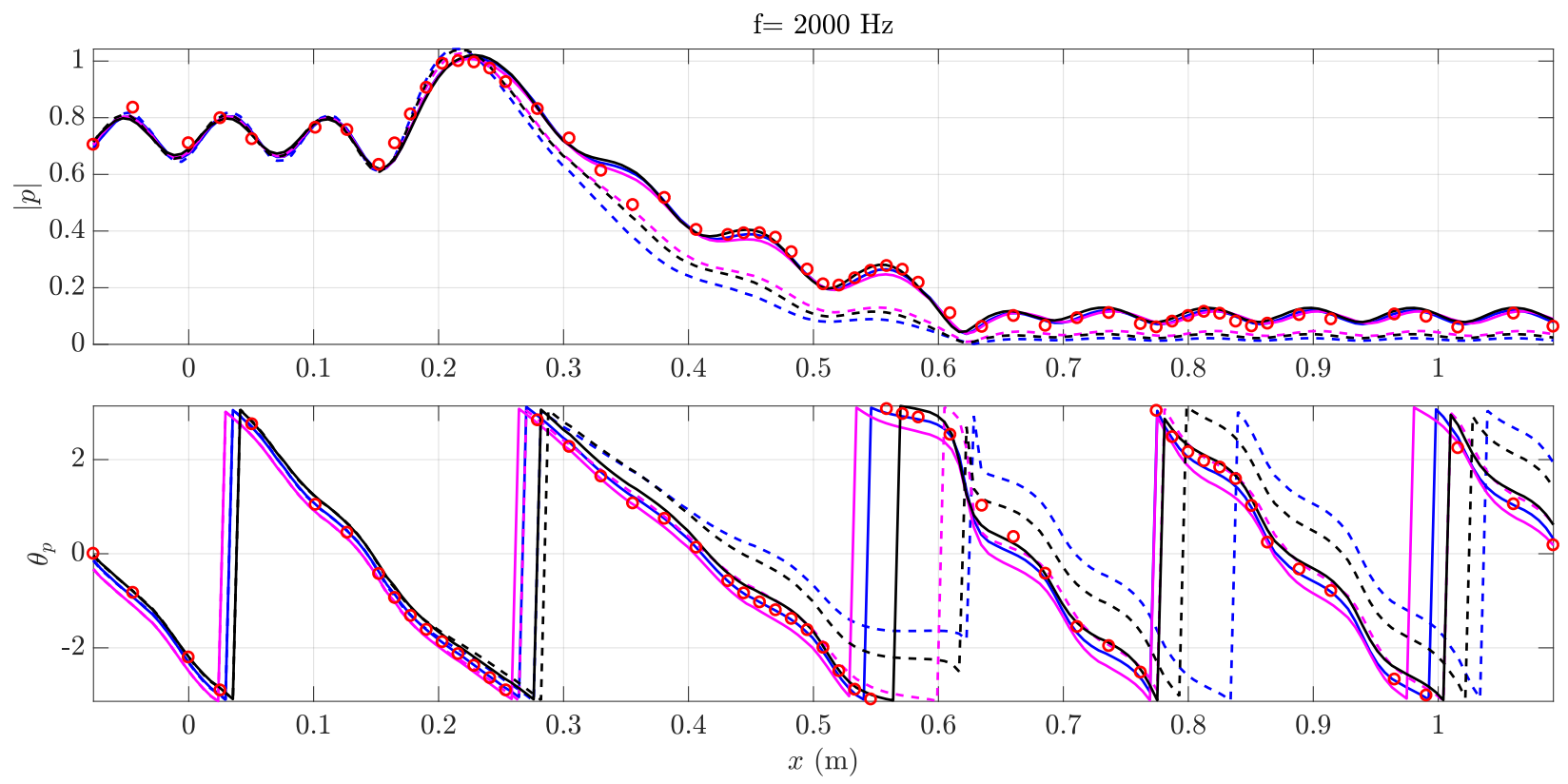

Figure 10 Wall pressure in upstream source configuration for the GE03. Upper: absolute pressure. Lower: phase. Symbols: experimental data.
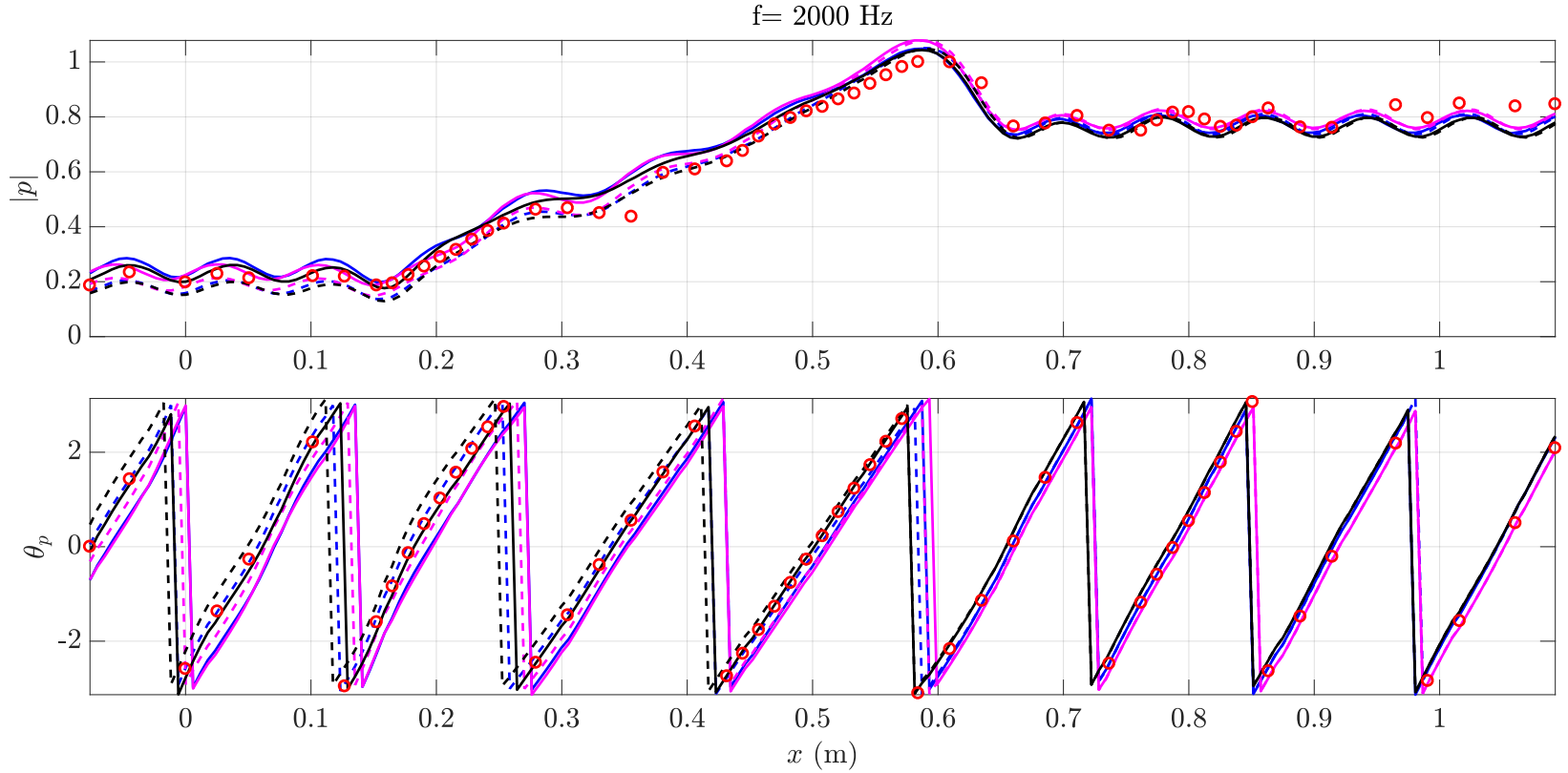

Figure 11 Wall pressure in downstream source configuration for the GE03. Upper: absolute pressure. Lower: phase. Symbols: experimental data.

The differences in the curves are more marked when the impedance is taken as the one educed with the opposite source location, as compared to the case where the flow profile is changed. However, as opposed to the CT57, the pressure sensitivity to a variation of $\zeta$ is more visible for the upstream configuration (Fig. 10) instead of the downstream one (Fig. 111). A quantitative analysis of the spread between curves is performed by assigning to each curve its cost function value (minus log of the likelihood) in Table 3, normalized by the minimum value obtained (0.05). 
Table 3 Comparison of the cost function values associated with Figs. 10 , for the GE03 at $2000 \mathrm{~Hz}$.

\begin{tabular}{ccccccc} 
& \multicolumn{3}{c}{ Upstream Identification } & \multicolumn{3}{c}{ Downstream Identification } \\
\hline & Flow 1 & Flow 2 & Flow 3 & Flow 1 & Flow 2 & Flow 3 \\
Educed impedance & $0.46+0 \mathrm{j}$ & $0.48-0.01 \mathrm{j}$ & $0.47+0 \mathrm{j}$ & $0.4-0.28 \mathrm{j}$ & $0.45-0.16 \mathrm{j}$ & $0.43-0.22 \mathrm{j}$ \\
Cost function in upstream conf. & 1 & 5.2 & 5.6 & 18.4 & 7.4 & 18.8 \\
Cost function in downstream conf. & 20.8 & 32.4 & 9.2 & 5 & 16.8 & 15.6
\end{tabular}

The fits obtained with a more sheared flow (flow 1, see Fig. 2) appear again to be of superior quality than the ones with less sheared profiles, in both source configurations. The fits are of higher quality in the upstream source configuration, where the cost function value is the lowest. Despite a much lower variation between educed impedance, the relative spread in cost function is comparable to the one obtained for the CT57 at the antiresonance. This is explained by a difference in the sensitivity of the cost function in the resonance and antiresonance frequency regions.

\section{Conclusions}

This study has shown that the difference in educed impedance when considering different source locations for the measurements could not be explained by a possible defect in the modeling of the tangential velocity profile. Changing the shear flow profile results in a similar change in educed impedance for both source locations. It does not improve the consistency between impedance curves, as was achieved by changing the bulk Mach number in [7].

\section{Acknowledgments}

This work is part of the NASA/ONERA collaborative agreement on aircraft noise reduction.

\section{References}

[1] Yves Aurégan, Maud Leroux, and Vincent Pagneux. Measurement of liner impedance with flow by an inverse method. In 10th AIAA/CEAS Aeroacoustics Conference, page 2838, 2004.

[2] T Elnady, Hans Bodén, and B Elhadidi. Validation of an inverse semi-analytical technique to educe liner impedance. AIAA journal, 47(12):2836-2844, 2009.

[3] Walter Eversman and Judith M Gallman. Impedance eduction with an extended search procedure. AIAA journal, 49(9):1960-1970, 2011.

[4] Xiaodong Jing, Sen Peng, and Xiaofeng Sun. A straightforward method for wall impedance eduction in a flow duct. The Journal of the Acoustical Society of America, 124(1):227-234, 2008.

[5] Michael G Jones, Willie R Watson, and Tony L Parrott. Benchmark data for evaluation of aeroacoustic propagation codes with grazing flow. AIAA paper, 2853:2005, 2005.

[6] Fabien Mery, Rémi Roncen, Estelle Piot, and Frank Simon. Statistical inference of liner impedance with shear grazing flow. In 2018 AIAA/CEAS Aeroacoustics Conference, page 3775, 2018.

[7] Douglas M Nark, Michael G Jones, and Estelle Piot. Assessment of axial wave number and mean flow uncertainty on acoustic liner impedance eduction. In 2018 AIAA/CEAS Aeroacoustics Conference, page 3444, 2018.

[8] L. Pascal, E. Piot, and G. Casalis. Discontinuous Galerkin method for the computation of acoustic modes in lined flow ducts with rigid splices. Journal of Sound and Vibration, 332(13):3270 - 3288, 2013.

[9] Lucas Pascal, Estelle Piot, and Grégoire Casalis. Discontinuous galerkin method for acoustic modes computation in lined ducts. In 18th AIAA/CEAS Aeroacoustics Conference (33rd AIAA Aeroacoustics Conference), page 2153, 2012.

[10] Estelle Piot, Julien Primus, and Frank Simon. Liner impedance eduction technique based on velocity fields. In 18 th AIAA/CEAS Aeroacoustics Conference (33rd AIAA Aeroacoustics Conference), page 2198, 2012.

[11] Julien Primus, Estelle Piot, and Frank Simon. An adjoint-based method for liner impedance eduction: Validation and numerical investigation. Journal of Sound and Vibration, 332(1):58-75, 2013. 
[12] Ygaäl Renou and Yves Aurégan. Failure of the ingard-myers boundary condition for a lined duct: An experimental investigation. The Journal of the Acoustical Society of America, 130(1):52-60, 2011.

[13] X Sun, X Jing, H Zhang, and Y Shi. Effect of grazing-bias flow interaction on acoustic impedance of perforated plates. $J$. Sound Vib., 254(3):557-573, 2002.

[14] Willie R Watson and Michael G Jones. A comparative study of four impedance eduction methodologies using several test liners. In 19th AIAA/CEAS Aeroacoustics Conference, page 2274, 2013.

[15] Chenyang Weng, Anita Schulz, Dirk Ronneberger, Lars Enghardt, and Friedrich Bake. Impedance eduction in the presence of turbulent shear flow using the linearized navier-stokes equations. In 23rd AIAA/CEAS Aeroacoustics Conference, page 3182 , 2017.

[16] Qi Zhang. Direct numerical investigation and reduced-order modeling of 3-D honeycomb acoustic liners. PhD thesis, University of Illinois at Urbana-Champaign, 2014.

[17] Lin Zhou, Hans Boden, Claus Lahiri, Friedrich Bake, Lars Enghardt, Stefan Busse-Gerstengarbe, and Tamer Elnady. Comparison of impedance eduction results using different methods and test rigs. In 20th AIAA/CEAS Aeroacoustics Conference, page 2955 , 2014. 